\title{
Rotating points for the conformal NLS scattering operator
}

\author{
Rémi Carles
}

Communicated by Terence Tao, received November 19, 2008.

\begin{abstract}
We consider the nonlinear Schrödinger equation, with mass-critical nonlinearity, focusing or defocusing. For any given angle, we establish the existence of infinitely many functions on which the scattering operator acts as a rotation of this angle. Using a lens transform, we reduce the problem to the existence of a solution to a nonlinear Schrödinger equation with harmonic potential, satisfying suitable periodicity properties. The existence of infinitely many such solutions is proved thanks to a constrained minimization problem.
\end{abstract}

\section{Contents}

1. Introduction

2. Reduction of the problem 39

3. Construction of periodic solutions 42

4. End of the proof of Theorem $1.8 \quad 45$

Appendix A. Profile decomposition and nonlinear superposition $\quad 47$ References

\section{Introduction}

We consider the pseudo-conformally invariant nonlinear Schrödinger equation

$$
i \partial_{t} u+\frac{1}{2} \Delta u=|u|^{4 / d} u, \quad(t, x) \in \mathbf{R} \times \mathbf{R}^{d}, d \geqslant 1 .
$$

Two types of initial data are of special interest:

$$
\begin{aligned}
& \text { Asymptotic state: }\left.U_{0}(-t) u(t)\right|_{t= \pm \infty}=u_{ \pm}, \quad \text { where } U_{0}(t)=e^{i \frac{t}{2} \Delta} \text {. } \\
& \text { Cauchy data at } t=0: u_{\mid t=0}=u_{0} .
\end{aligned}
$$

1991 Mathematics Subject Classification. 35.

Key words and phrases. Rotating points, nonlinear Schrödinger equation, scattering operator, harmonic potential.

This work was supported by the French ANR project R.A.S. (ANR-08-JCJC-0124-01). 
It is well known that for data $u_{ \pm} \in \Sigma=H^{1} \cap \mathcal{F}\left(H^{1}\right)$, where

$$
\mathcal{F} f(\xi)=\widehat{f}(\xi)=\frac{1}{(2 \pi)^{d / 2}} \int_{\mathbf{R}^{d}} f(x) e^{-i x \cdot \xi} d x,
$$

(1.1)-(1.2) has a unique, global, solution $u \in C(\mathbf{R} ; \Sigma)$ ([17], see also [10]). Its initial value $u_{\mid t=0}$ is the image of the asymptotic state under the action of the wave operator:

$$
u_{\mid t=0}=W_{ \pm} u_{ \pm}
$$

Similarly, if $u_{0} \in \Sigma$, (1.1)-(1.3) possesses asymptotic states:

$$
\exists u_{ \pm} \in \Sigma, \quad\left\|U_{0}(-t) u(t)-u_{ \pm}\right\|_{\Sigma} \underset{t \rightarrow \pm \infty}{\longrightarrow} 0: \quad u_{ \pm}=W_{ \pm}^{-1} u_{0} .
$$

The scattering operator associated to (1.1) is classically defined as

$$
S=W_{+}^{-1} \circ W_{-}: u_{-} \mapsto u_{+} .
$$

It maps $\Sigma$ to $\Sigma$, and is unitary on $L^{2}$, and on $\dot{H}^{1}$ :

$$
\left\|S\left(u_{-}\right)\right\|_{L^{2}\left(\mathbf{R}^{d}\right)}=\left\|u_{-}\right\|_{L^{2}\left(\mathbf{R}^{d}\right)} \quad ; \quad\left\|\nabla S\left(u_{-}\right)\right\|_{L^{2}\left(\mathbf{R}^{d}\right)}=\left\|\nabla u_{-}\right\|_{L^{2}\left(\mathbf{R}^{d}\right)} .
$$

This follows from $[17,38,18]$.

Besides the existence of the wave and scattering operators, it seems that very few of their properties are known. By construction, these operators are continuous on $\Sigma$. When $d \leqslant 2$ (the nonlinearity is smooth), these operators are real analytic on $\Sigma$; see $[7]$.

It is rather reassuring to check that the operators $W_{ \pm}$and $S$ are not trivial, showing that averaged in time nonlinear effects may not be negligible. Following [15] for the case of the wave equation, we can prove for instance that in $L^{2}\left(\mathbf{R}^{d}\right)$, and as $\varepsilon \rightarrow 0$,

$$
S\left(\varepsilon u_{-}\right)=\varepsilon u_{-}-i \varepsilon^{1+4 / d} P\left(u_{-}\right)+\mathcal{O}\left(\varepsilon^{1+8 / d}\right)
$$

where

$$
P\left(u_{-}\right)=\int_{-\infty}^{+\infty} U_{0}(-t)\left(\left|U_{0}(t) u_{-}\right|^{4 / d} U_{0}(t) u_{-}\right) d t .
$$

We refer to [9] for a proof (in the present small data case, it suffices to assume that $\left.u_{-} \in L^{2}\left(\mathbf{R}^{d}\right)\right)$. Explicit computations show that $P\left(u_{-}\right) \neq 0$ when $u_{-}$is Gaussian, therefore $S$ is not the identity.

A few algebraic properties are available. Let $\mathcal{C}$ denote the conjugation $f \mapsto \bar{f}$. The invariances of the equation show that

$$
W_{ \pm}=\mathcal{C} \circ W_{\mp} \circ \mathcal{C}
$$

an identity which was noticed in [12] (see also [10]). Due to the invariance of (1.1) under translation and gauge transforms,

$$
\begin{aligned}
& S\left(u_{-}(\cdot+a)\right)=S\left(u_{-}\right)(\cdot+a), \forall a \in \mathbf{R}^{d}, \\
& S\left(e^{i \eta} u_{-}\right)=e^{i \eta} S\left(u_{-}\right), \forall \eta \in \mathbf{R} .
\end{aligned}
$$

Another algebraic relation was established in [9], which seems to be bound to the conformal case, contrary to (1.6) and (1.7):

$$
\mathcal{F} \circ W_{ \pm}^{-1}=W_{\mp} \circ \mathcal{F} .
$$


In [1], a remarkable property was proved for the scattering operator associated to the energy-critical wave equation

$$
\partial_{t}^{2} u-\Delta u+|u|^{4} u=0 \quad ; \quad x \in \mathbf{R}^{3} .
$$

Using the notion of profile decomposition, as introduced in [16, 29], the authors prove in [1] that the scattering operator associated to (1.8), and defined on the energy space, enjoys a surprising nonlinear superposition principle. It follows from $[21,14]$ that a similar result holds for the Schrödinger analogue of (1.8),

$$
i \partial_{t} u+\frac{1}{2} \Delta u=|u|^{4} u \quad ; \quad x \in \mathbf{R}^{3} .
$$

We give more precise statements in an appendix, where we also discuss the case of (1.1). In this paper, we show the existence of infinitely many fixed points for $S$, and more generally:

THEOREM 1.1. Let $d \geqslant 1$ and $S: \Sigma \rightarrow \Sigma$ be the scattering operator associated to (1.1). For any $\theta \in\left[0,2 \pi\left[\right.\right.$, there are infinitely many functions $u_{-} \in \Sigma$ such that $S\left(u_{-}\right)=e^{i \theta} u_{-}$.

REMARK 1.2. For $\theta \in] 0,2 \pi[$, this result yields another evidence that $S$ is not trivial.

REMARK 1.3. For $\theta=0$, this shows the existence of infinitely many fixed points. Similarly, for $\theta=2 \pi p / q, p, q \in \mathbf{N}^{*}$, this shows the existence of infinitely many periodic (or cyclic) points, for any given period.

REMARK 1.4. We construct solutions of the form

$$
u(t, x)=\frac{1}{\left(1+t^{2}\right)^{d / 4}} e^{i \frac{t}{1+t^{2}} \frac{|x|^{2}}{2}-i\left(\frac{d}{2}+2 j-\frac{\theta}{\pi}\right) \arctan t} \phi_{j}\left(\frac{x}{\sqrt{1+t^{2}}}\right),
$$

where $j \in \mathbf{N} \backslash\{0\}$ and $\phi_{j} \in \Sigma$. The profile $\phi_{j}$ is given by the nonlinear eigenvalue equation (3.1) with $\nu=d / 2+2 j+\theta / \pi$.

REMARK 1.5. In semi-classical analysis, the scattering operator appears in some cases to describe solutions which pass through a focal point. In the presence of an isotropic (but not necessarily) harmonic potential, focusing at one point occurs periodically in time, and the scattering operator is iterated each time a focal point is traversed; see [6] and references therein. The existence of fixed points, and more generally, of periodic points, shows that the nonlinear dynamics may reveal some periodicity in time, at leading order in the semi-classical limit.

REMARK 1.6. Not all the functions in $\Sigma$ are such that $S\left(u_{-}\right)(x)=e^{i h(x)} u_{-}(x)$ for some real-valued function $h$ (not necessarily constant). Arguing by contradiction and using (1.5), one can show that there exist two functions $u_{-}$and $\widetilde{u}_{-}$in $\Sigma$ such that

See $[6, \S 7.4 .3]$.

$$
\left|u_{-}(x)\right| \equiv\left|\widetilde{u}_{-}(x)\right|, \quad \text { and } \quad\left|S\left(u_{-}\right)(x)\right| \not \equiv\left|S\left(\widetilde{u}_{-}\right)(x)\right| .
$$

REMARK 1.7. For the linear Schrödinger equation, one can construct transparent potentials $V(x)$. This means that one can choose a potential $V$ such that any function $u(t, x)=e^{i E t} \psi(x), E \in \mathbf{R}$, solution to

$$
i \partial_{t} u+\frac{1}{2} \Delta u=V u
$$

has a trivial scattering matrix; see [23] and references therein. 
In the focusing case

$$
i \partial_{t} u+\frac{1}{2} \Delta u=-|u|^{4 / d} u, \quad(t, x) \in \mathbf{R} \times \mathbf{R}^{d},
$$

no general scattering theory is available, since finite time blow-up may occur (see e.g. $[10,36]$ ). We know however that for (initial or asymptotic) data with a sufficiently small $L^{2}$ norm, the solution is global, and there is scattering [11]. Recall that the ground state given as the unique positive, radially symmetric solution to

$$
-\frac{1}{2} \Delta Q+Q=Q^{1+4 / d}
$$

yields the best constant for the following Gagliardo-Nirenberg inequality [41]:

$$
\|f\|_{L^{2+4 / d}}^{2+4 / d} \leqslant \frac{d+2}{2 d\|Q\|_{L^{2}}^{4 / d}}\|f\|_{L^{2}}^{4 / d}\|\nabla f\|_{L^{2}}^{2}, \quad \forall f \in H^{1}\left(\mathbf{R}^{d}\right) .
$$

If $\|u\|_{L^{2}}<\|Q\|_{L^{2}}$, then all $H^{1}$-solutions to (1.9) are global in time [41]. It is conjectured that the same holds true for $L^{2}$-solutions; see [27, 22, 19, 39, 40, 24, $37,25]$, and references therein.

THEOREM 1.8. Let $d \geqslant 1$. For any $\theta \in[0,2 \pi[$, there are infinitely many functions $u_{-} \in \Sigma$ such that the scattering operator associated to the focusing equation (1.9) is well defined on $u_{-}$, and such that $S\left(u_{-}\right)=e^{i \theta} u_{-}$. In addition,

- These functions satisfy $\left\|u_{-}\right\|_{L^{2}}>\left(\frac{d}{d+2}\right)^{d / 4}\|Q\|_{L^{2}}$.

- They are arbitrarily large in $H^{1}\left(\mathbf{R}^{d}\right)$ (resp. in $\dot{H}^{1}\left(\mathbf{R}^{d}\right)$ if $d \geqslant 2$ ).

- For all such $u_{-} \in \Sigma$, there exists $\varepsilon>0$ such that if $\widetilde{u}_{-} \in \Sigma$ satisfies $\left\|u_{-}-\widetilde{u}_{-}\right\|_{L^{2}}<\varepsilon$, then $S\left(\widetilde{u}_{-}\right)$is well-defined in $\Sigma$ (in particular, there is no blow-up).

REMARK 1.9. We construct solutions of the same form as in Remark 1.4, but with different profiles $\phi$, and in the phase, $-j \in \mathbf{N}$, with $-2 j>d / 2$.

REMARK 1.10. In the defocusing case, the last property stated above is straightforward, since $S$ is defined on $\Sigma$. In the above focusing case, this stability property is more surprising.

Theorems 1.1 and 1.8 rely on two steps. As shown in $\S 2$, a lens transform reduces the proof to the existence of time periodic solutions for the equation

$$
i \partial_{t} v+\frac{1}{2} \Delta v=\frac{|x|^{2}}{2} v \pm|v|^{4 / d} v
$$

Since the nonlinearity is autonomous, it is reasonable to expect solutions to the above equation which are standing waves, $v(t, x)=e^{-i \nu t} \psi(x)$. The point is that infinitely many values for $\nu$ lead to periodic solutions with a suitable period, that is, such that by inverting the lens transform, we get Theorem 1.1 and the existence part of Theorem 1.8. This step is achieved in $\S 3$. The rest of the proof of Theorem 1.8 is given in $\S 4$. Finally, in the appendix, we discuss the nonlinear superposition principle associated to the scattering operator for (1.1), modulo some global existence issues which are still open so far. 


\section{Reduction of the problem}

2.1. Lens transform. Let $u \in C(\mathbf{R} ; \Sigma)$ solve the more general equation

$$
i \partial_{t} u+\frac{1}{2} \Delta u=|u|^{2 \sigma} u, \quad(t, x) \in \mathbf{R} \times \mathbf{R}^{d} .
$$

If

$$
\begin{aligned}
& \sigma_{0}(d)<\sigma<\frac{2}{d-2} \quad\left(\text { with only } \sigma>\sigma_{0}(d) \text { if } d \leqslant 2\right), \\
& \text { where } \sigma_{0}(d):=\frac{2-d+\sqrt{d^{2}+12 d+4}}{4 d},
\end{aligned}
$$

then the scattering operator associated to (2.1) is well defined, from $\Sigma$ to $\Sigma$; [17, $38,18]$ (see also $[12,30]$ where the case $\sigma=\sigma_{0}(d)$ is allowed). Introduce

$$
v(t, x)=\frac{1}{(\cos t)^{d / 2}} u\left(\tan t, \frac{x}{\cos t}\right) e^{-i \frac{|x|^{2}}{2} \tan t},
$$

which is well defined for $|t|<\pi / 2$, and has the same value as $u$ at time $t=0$. As noticed in $[20,34]$ (see also $[4,35]), v$ solves, at least formally:

$$
i \partial_{t} v+\frac{1}{2} \Delta v=\frac{|x|^{2}}{2} v+|\cos t|^{d \sigma-2}|v|^{2 \sigma} v
$$

Note an important feature of the lens transform (2.3): it maps the line $\mathbf{R}_{t}$ for $u$, to the bounded interval $]-\frac{\pi}{2}, \frac{\pi}{2}$ [ for $v$. Therefore, long time properties for $u$ are equivalent to local in time properties for $v$.

\subsection{The harmonic oscillator. Let}

$$
H=-\frac{1}{2} \Delta+\frac{|x|^{2}}{2}, \text { and } U_{H}(t)=e^{-i t H}
$$

denote the harmonic oscillator and its propagator. Recall some well-known properties (see e.g. [26]):

LEMMA 2.1. We have

$$
\sigma_{p}(H)=\left\{\frac{d}{2}+k=: l_{k} ; k \in \mathbf{N}\right\},
$$

and the associated eigenfunctions are given by (tensor products of) Hermite functions, which form a basis of $L^{2}\left(\mathbf{R}^{d}\right)$. For $\ell \in \mathbf{N}$, the eigenfunctions associated to $l_{2 \ell}\left(\right.$ resp. $\left.l_{2 \ell+1}\right)$ are even (resp. odd).

In space dimension $d=1$, these eigenvalues are simple, and the eigenvectors are given by Hermite functions $\left(\psi_{k}\right)_{k \in \mathbf{N}}$. For even indices, $\psi_{2 \ell}$ is even, and for odd indices, $\psi_{2 \ell+1}$ is odd. Up to normalizing constants, we have for instance

$$
\psi_{0}(x)=e^{-x^{2} / 2} \quad ; \quad \psi_{1}(x)=x e^{-x^{2} / 2} .
$$

In higher dimensions, one considers tensor products of one-dimensional eigenfunctions; the eigenvalues $l_{k}>d / 2$ are no longer simple. We note the identity

$$
U_{H}(t+\pi) \psi(x)=e^{-i d \pi / 2} U_{H}(t) \psi(-x), \quad \forall t \in \mathbf{R} .
$$

Finally, as a direct consequence of Mehler's formula, local in time Strichartz estimates are available for $U_{H}$ (see e.g. $[5,10]$ ). Notice that since $H$ possesses eigenvalues, global in time Strichartz estimates fails for $U_{H}$. 
Lemma 2.2. Let $d \geqslant 1$. A pair $(p, q)$ is admissible provided that

$$
\frac{2}{p}+\frac{d}{q}=\frac{d}{2}, \quad p \geqslant 2, \quad(p, q, d) \neq(2, \infty, 2) .
$$

Consider a finite time interval $I$.

1. For all admissible pair $(p, q)$, there exists $C=C(p,|I|)$ such that

$$
\left\|U_{H}(\cdot) \phi\right\|_{L^{p}\left(I ; L^{q}\left(\mathbf{R}^{d}\right)\right)} \leqslant C\|\phi\|_{L^{2}}, \quad \forall \phi \in L^{2}\left(\mathbf{R}^{d}\right) .
$$

2. Define the retarded operator in defined by

$$
R(F)(t, x)=\int_{I \cap\{s \leqslant t\}} U_{H}(t-s) F(s, x) d s .
$$

For all admissible pairs $\left(p_{1}, q_{1}\right),\left(p_{2}, q_{2}\right)$, there exists $C=C\left(p_{1}, p_{2},|I|\right)$ with

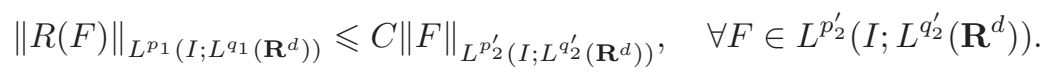

2.3. A rotating point for $S$. The following lemma is standard (see [38] or [32]):

LEMMA 2.3. Let $f \in L^{2}\left(\mathbf{R}^{d}\right)$, and recall that $U_{0}(t)=e^{i \frac{t}{2} \Delta}$.

$$
\left\|U_{0}(t) f-A(t) f\right\|_{L^{2}\left(\mathbf{R}^{d}\right)} \underset{t \rightarrow \pm \infty}{\longrightarrow} 0, \quad \text { where } A(t) f(x)=\frac{1}{(i t)^{d / 2}} \widehat{f}\left(\frac{x}{t}\right) e^{i \frac{|x|^{2}}{2 t}},
$$

and the Fourier transform is normalized in (1.4).

Proof. From the explicit formula

$$
U_{0}(t) f(x)=\frac{1}{(2 i \pi t)^{d / 2}} \int_{\mathbf{R}^{d}} e^{i \frac{|x-y|^{2}}{2 t}} f(y) d y,
$$

we have the following factorization:

$$
U_{0}(t)=M_{t} D_{t} \mathcal{F} M_{t},
$$

where $M_{t}$ stands for the multiplication by the function $e^{i \frac{|x|^{2}}{2 t}}, \mathcal{F}$ is the Fourier transform defined in (1.4), and $D_{t}$ is the dilation operator

$$
\left(D_{t} f\right)(x)=\frac{1}{(i t)^{d / 2}} f\left(\frac{x}{t}\right) \text {. }
$$

The lemma thus reads: $U_{0}(t)-M_{t} D_{t} \mathcal{F} \rightarrow 0$ strongly in $L^{2}$, as $t \rightarrow \pm \infty$. Since $M_{t} D_{t} \mathcal{F}$ is unitary on $L^{2}$, the lemma follows from the strong limit in $L^{2}, M_{t}-\mathrm{Id} \rightarrow 0$, which stems from the Dominated Convergence Theorem.

LEMmA 2.4. Let $\sigma$ satisfy (2.2), and $u, v \in C(\mathbf{R} ; \Sigma)$ solve (2.1) and (2.4), respectively, with $u_{\mid t=0}=v_{\mid t=0}=\phi$. Then

$$
v\left(-\frac{\pi}{2}, x\right)=e^{i d \pi / 4} \mathcal{F}\left(W_{-}^{-1} \phi\right)(-x) \quad ; \quad v\left(\frac{\pi}{2}, x\right)=e^{-i d \pi / 4} \mathcal{F}\left(W_{+}^{-1} \phi\right)(x) .
$$

REMARK 2.5. In the linear case, the same result holds when $W_{ \pm}$are replaced by Id: see (2.5). The $-d \pi / 2$ phase shift between the two instants $\pm \pi / 2$ corresponds to the Maslov index, and the symmetry with respect to the origin accounts for the fact that the harmonic oscillator rotates the phase space with angular velocity equal to one. 
Remark 2.6. It would suffice to consider $v \in C\left([-\pi / 2, \pi / 2] ; L^{2}\left(\mathbf{R}^{d}\right)\right)$, and $u \in C\left(\mathbf{R} ; L^{2}\left(\mathbf{R}^{d}\right)\right)$ which has asymptotic states in $L^{2}\left(\mathbf{R}^{d}\right)$. This is guaranteed if we assume further spatial regularity as above. In the case $\sigma=2 / d$, we might also consider either data with small $L^{2}$ norm [11], or radially symmetric $L^{2}$ functions when $d \geqslant 2[24,37]$. However, for the construction of periodic solutions to (2.4), we take advantage of properties such as the compactness of the embedding $\Sigma \hookrightarrow$ $L^{2} \cap L^{2 \sigma+2}$.

Proof. We show:

$$
\left\|v(t)-e^{-i d \pi / 4} \mathcal{F}\left(W_{+}^{-1} \phi\right)\right\|_{L^{2}\left(\mathbf{R}^{d}\right)} \rightarrow 0 \text { as } t \underset{<}{<} .
$$

By (2.3) and asymptotic completeness for (1.1), we have, in $L^{2}\left(\mathbf{R}^{d}\right)$ :

$$
\begin{aligned}
& v(t, x)=\frac{1}{(\cos t)^{d / 2}} e^{-i \frac{|x|^{2}}{2} \tan t} u\left(\tan t, \frac{x}{\cos t}\right) \\
& \underset{t \rightarrow \frac{\pi}{2}^{-}}{\sim} \frac{1}{(\cos t)^{d / 2}} e^{-i \frac{|x|^{2}}{2} \tan t}\left(U_{0}(\tan t) u_{+}\right)\left(\frac{x}{\cos t}\right),
\end{aligned}
$$

where $u_{+}=W_{+}^{-1} \phi$. By Lemma 2.3, we infer

$$
v(t, x) \underset{t \rightarrow \frac{\pi}{2}-}{\sim} \frac{1}{(\cos t)^{d / 2}} e^{-i \frac{|x|^{2}}{2} \tan t} e^{i\left|\frac{x}{\cos t}\right|^{2} \frac{1}{2 \tan t}} \frac{1}{(i \tan t)^{d / 2}} \widehat{u}_{+}\left(\frac{x}{\tan t \cos t}\right) .
$$

The last quantity is equal to

$$
\frac{e^{-i d \pi / 4}}{(\sin t)^{d / 2}} \widehat{u}_{+}\left(\frac{x}{\sin t}\right) e^{i \frac{|x|^{2}}{2}\left(\frac{1}{\cos t \sin t}-\frac{\sin t}{\cos t}\right)},
$$

which converges to $e^{-i d \pi / 4} \widehat{u}_{+}(\cdot)$ in $L^{2}\left(\mathbf{R}^{d}\right)$ as $t \rightarrow \pi / 2$.

The case $t \rightarrow-\pi / 2$ is similar, up to a symmetry with respect to the origin, since $\sin (-\pi / 2)=-1$.

Denote $u_{-}=W_{-}^{-1} \phi$. We have therefore

$$
v\left(-\frac{\pi}{2}, x\right)=e^{+i d \pi / 4} \mathcal{F}\left(u_{-}\right)(-x) \quad ; \quad v\left(\frac{\pi}{2}, x\right)=e^{-i d \pi / 4} \mathcal{F}\left(S u_{-}\right)(x) .
$$

ConClusion. If the solution $v$ to (2.4) with $v_{\mid t=0}=\phi$ satisfies

$$
v\left(\frac{\pi}{2}, x\right)=e^{-i d \pi / 2+i \theta} v\left(-\frac{\pi}{2},-x\right),
$$

then $u_{-}=W_{-}^{-1} \phi$ verifies $S\left(u_{-}\right)=e^{i \theta} u_{-}$.

2.4. More rotating points? In the case $\sigma=2 / d$, the nonlinearity in (2.4) is autonomous: we may apply the lens transform back and forth, and change the time origin. The following result is then straightforward:

Proposition 2.7. Let $d \geqslant 1$ and $\sigma=2 / d$. Let $v \in C(\mathbf{R} ; \Sigma)$ solve (2.4), and such that

$$
v(t+\pi, x)=e^{-i d \pi / 2+i \theta} v(t,-x), \quad \forall(t, x) \in \mathbf{R} \times \mathbf{R}^{d} .
$$

Then for all $t \in \mathbf{R}, v(t, \cdot) \in \Sigma$ is such that $u_{-}^{t}=W_{-}^{-1} v(t)$ satisfies

$$
S\left(u_{-}^{t}\right)=e^{i \theta} u_{-}^{t} .
$$

REMARK 2.8. Due to the gauge invariance of (1.1), $S$ is also gauge invariant - see (1.7) - and the above result may be relevant only on time intervals of length (at most) $\pi$. 
2.5. The focusing case. Consider the equation:

$$
i \partial_{t} v+\frac{1}{2} \Delta v=\frac{|x|^{2}}{2} v-|v|^{4 / d} v .
$$

For initial data in $\Sigma$, the existence of a unique solution locally in time is well-known (see e.g. [10]). Rather than the possibility of finite time blow-up, our interest is:

Lemma 2.9. Suppose that $(2.7)$ has a solution $v \in C([-\pi / 2, \pi / 2] ; \Sigma)$. Then $u$, defined by

$$
u(t, x)=\frac{1}{\left(1+t^{2}\right)^{d / 4}} e^{i \frac{t}{1+t^{2}} \frac{|x|^{2}}{2}} v\left(\arctan t, \frac{x}{\sqrt{1+t^{2}}}\right)
$$

solves (1.9). It satisfies $u \in C(\mathbf{R} ; \Sigma)$, and has asymptotic states in $\Sigma$, given by:

$$
u_{ \pm}(x)=e^{ \pm i d \pi / 4} \mathcal{F}^{-1} v\left( \pm \frac{\pi}{2}\right)( \pm x) .
$$

Proof. We have immediately $u \in C(\mathbf{R} ; \Sigma)$, and the fact that it solves (1.9). To see that $u$ has asymptotic states, given by the above formula, write, for large $|t|:$

$$
\begin{aligned}
& U_{0}(-t) u(t, x)=\frac{1}{(-2 i \pi t)^{d / 2}} \int e^{-i \frac{|x-y|^{2}}{2 t}} u(t, y) d y \\
& =\frac{1}{(-2 i \pi t)^{d / 2}} \int \frac{1}{\left(1+t^{2}\right)^{d / 4}} e^{i \frac{t}{1+t^{2}} \frac{|y|^{2}}{2}} e^{-i \frac{|x-y|^{2}}{2 t}} v\left(\arctan t, \frac{y}{\sqrt{1+t^{2}}}\right) d y \\
& \approx \frac{1}{(-2 i \pi t)^{d / 2}} \frac{1}{|t|^{d / 2}} e^{-i \frac{|x|^{2}}{2 t}} \int e^{i \frac{x \cdot y}{t}} e^{i\left(-\frac{1}{t}+\frac{t}{1+t^{2}}\right) \frac{|y|^{2}}{2}} v\left(\arctan t, \frac{y}{\sqrt{1+t^{2}}}\right) d y \\
& \approx \frac{e^{ \pm i d \pi / 4}}{(2 \pi)^{d / 2}} \frac{1}{|t|^{d}} e^{-i \frac{|x|^{2}}{2 t}} \int e^{i \frac{x \cdot y}{t}} e^{-\frac{i}{t\left(1+t^{2}\right)} \frac{|y|^{2}}{2}} v\left(\arctan t, \frac{y}{|t|}\right) d y \\
& \approx e^{ \pm i d \pi / 4} e^{-i \frac{|x|^{2}}{2 t}} \mathcal{F}^{-1} v(\arctan t, \pm x) \approx e^{ \pm i d \pi / 4} \mathcal{F}^{-1} v\left( \pm \frac{\pi}{2}, \pm x\right) .
\end{aligned}
$$

We have presented the computations in a formal way. We leave their easy justification to the reader.

\section{Construction of periodic solutions}

We construct a solution of the form

$$
v(t, x)=e^{-i \nu t} \psi(x) .
$$

In the defocusing case, $\psi$ must solve

$$
\nu \psi=H \psi+|\psi|^{4 / d} \psi .
$$

In the focusing case, it must solve

$$
(H-\nu) \psi=|\psi|^{4 / d} \psi
$$

We have:

Proposition 3.1. Let $d \geqslant 1$.

1. If $\nu>d / 2$, then there exists an even function $\psi \in \Sigma \backslash\{0\}$ solving (3.1).

2. If $\nu<d / 2$, then there exists an even function $\psi \in \Sigma \backslash\{0\}$ solving (3.2). 
Theorem 1.1 follows from the first point, by considering the family (for $\theta \in$ $[0,2 \pi[)$

$$
\left(\nu_{j}\right)_{j \geqslant 1}=\left\{\frac{d}{2}+2 j-\frac{\theta}{\pi}, j \in \mathbf{N} \backslash\{0\}\right\} .
$$

The form of the corresponding solution $u$ given in Remark 1.4 is straightforward, by inverting the lens transform (2.3) (see (2.8)).

To infer the existence part of Theorem 1.8, we can consider the family (for $\theta \in[0,2 \pi[)$

$$
\left(\nu_{j}\right)_{j \geqslant 1}=\left\{\frac{d}{2}-2 j-\frac{\theta}{\pi}, j \in \mathbf{N} \backslash\{0\}\right\} .
$$

Remark 3.2. For such solutions, Proposition 2.7 is irrelevant. Consider $e^{-i \nu_{j} t} \psi(x)$ at two different times: this amounts to a multiplication by $e^{i \eta}$ for some $\eta \in \mathbf{R}$. As we have seen, the gauge invariance of (1.1) implies that $S$ is also gauge invariant, and Proposition 2.7 holds trivially for such solutions.

Remark 3.3. Proposition 3.1 remains valid if $|\psi|^{4 / d} \psi$ is replaced with $|\psi|^{2 \sigma} \psi$, where the nonlinearity is $H^{1}$-subcritical, that is, $\sigma<2 /(d-2)$ when $d \geqslant 3$. However, since the nonlinearity in (2.4) is autonomous if and only if $\sigma=2 / d$, this is the only case where it is reasonable to seek a solution to (2.4) of the form $v(t, x)=e^{-i \nu t} \psi(x)$.

At least two proofs of Proposition 3.1 are available in the literature:

- In [33], the case of (3.2) is considered, by using bifurcation theory. As indicated there, the arguments presented in [31] make it possible to infer Proposition 3.1.

- In [20], Proposition 3.1 is established up to the symmetry property (which could easily be incorporated): see [20, Theorem 1.4] for the first point, and [20, Theorem 1.3] for the second one. The proof there is based on the mountain pass lemma.

Even though this result has been established elsewhere, we present a another short, self-contained proof, for the sake of completeness.

Proof of Proposition 3.1. We proceed in the same spirit as in [3, 13]: let

$$
\begin{aligned}
I(\psi) & =\frac{1}{2}\langle H \psi, \psi\rangle-\frac{\nu}{2}\langle\psi, \psi\rangle, \\
M & =\left\{\psi \in \Sigma, \psi(x)=\psi(|x|) ; \frac{1}{1+2 / d} \int_{\mathbf{R}^{d}}|\psi(x)|^{2+4 / d} d x=1\right\} .
\end{aligned}
$$

We consider radially symmetric functions for simplicity; in particular, these are even functions. The following lines essentially show that the negative part of $I$ can be controlled. Denote

$$
\delta=\inf _{\psi \in M} I(\psi)
$$

First case: $\nu>d / 2$

We show that $0>\delta>-\infty$. To see that $\delta<0$, consider $\psi(x)=c e^{-|x|^{2} / 2}$, where $c$ is such that $\psi \in M$, and recall that $\psi$ is the unique eigenfunction associated to $l_{0}=d / 2: H \psi=d / 2 \psi$.

Suppose that we could find sequences in $M$ along which $I$ goes to $-\infty$. Let $\left(\psi_{n}\right)_{n \in \mathbf{N}}$ be such a sequence; necessarily, it is unbounded in $L^{2}\left(\mathbf{R}^{d}\right)$. We remark that $\left(x \psi_{n}\right)_{n \in \mathbf{N}}$ and $\left(\nabla \psi_{n}\right)_{n \in \mathbf{N}}$ are also unbounded in $L^{2}\left(\mathbf{R}^{d}\right)$, with norms of the 
same order as $\left\|\psi_{n}\right\|_{L^{2}}$. To be more precise, we introduce a notation: let $\left(\alpha_{n}\right)_{n \in \mathbf{N}}$ and $\left(\beta_{n}\right)_{n \in \mathbf{N}}$ be two families of positive real numbers.

- We write $\alpha_{n} \ll \beta_{n}$ if $\limsup _{n \rightarrow+\infty} \alpha_{n} / \beta_{n}=0$.

- We write $\alpha_{n} \lesssim \beta_{n}$ if $\limsup _{n \rightarrow+\infty} \alpha_{n} / \beta_{n}<\infty$.

- We write $\alpha_{n} \approx \beta_{n}$ if $\alpha_{n} \lesssim \beta_{n}$ and $\beta_{n} \lesssim \alpha_{n}$.

Let $\phi_{n} \in \Sigma$, with $\left\|\phi_{n}\right\|_{L^{2}} \rightarrow+\infty$. In general, up to extracting a subsequence, two possibilities can be distinguished:

- $\left\|\nabla \phi_{n}\right\|_{L^{2}} \gg\left\|\phi_{n}\right\|_{L^{2}}$ and/or $\left\|x \phi_{n}\right\|_{L^{2}} \gg\left\|\phi_{n}\right\|_{L^{2}}$,

- $\operatorname{Or}\left\|\nabla \phi_{n}\right\|_{L^{2}} \approx\left\|x \phi_{n}\right\|_{L^{2}} \approx\left\|\phi_{n}\right\|_{L^{2}}$.

This stems from the uncertainty principle

$$
\|\phi\|_{L^{2}}^{2} \leqslant \frac{2}{d}\|\nabla \phi\|_{L^{2}}\|x \phi\|_{L^{2}} .
$$

In our case, it is easy to see that the first possibility leads to a contradiction, since $I\left(\psi_{n}\right)$ would be positive for $n$ sufficiently large. Consider the last possible case: $\left\|\nabla \psi_{n}\right\|_{L^{2}} \approx\left\|x \psi_{n}\right\|_{L^{2}} \approx\left\|\psi_{n}\right\|_{L^{2}}$. Introduce

$$
\widetilde{\psi}_{n}=\frac{1}{\left\|\psi_{n}\right\|_{L^{2}}} \psi_{n}
$$

This is a bounded sequence in $\Sigma$, whose $L^{2}$ norm is equal to one. Up to extracting a subsequence, $\widetilde{\psi}_{n}$ converges weakly in $\Sigma$. Since $\Sigma \hookrightarrow L^{2}\left(\mathbf{R}^{d}\right)$ is compact, (a subsequence of) $\widetilde{\psi}_{n}$ converges strongly in $L^{2}\left(\mathbf{R}^{d}\right)$, to some $\widetilde{\psi} \in \Sigma$ such that $\|\widetilde{\psi}\|_{L^{2}}=1$. Since the embedding $\Sigma \hookrightarrow L^{2+4 / d}\left(\mathbf{R}^{d}\right)$ is compact, $\widetilde{\psi}_{n} \rightarrow \widetilde{\psi}$ strongly in $L^{2+4 / d}\left(\mathbf{R}^{d}\right)$. We infer

$$
\left\|\psi_{n}\right\|_{L^{2+4 / d}}=\left\|\psi_{n}\right\|_{L^{2}}\left\|\widetilde{\psi}_{n}\right\|_{L^{2+4 / d}} \approx\left\|\psi_{n}\right\|_{L^{2}} \rightarrow+\infty .
$$

Therefore, $\psi_{n}$ cannot remain in $M$, hence the finiteness of $\delta$.

Since the embedding $\Sigma \hookrightarrow L^{p}\left(\mathbf{R}^{d}\right)$ is compact for $2 \leqslant p<2 d /(d-2)(2 \leqslant p \leqslant \infty$ if $d=1$ and $2 \leqslant p<\infty$ if $d=2$ ), we infer that this infimum is actually a minimum, attained by a non-trivial function $\psi \in \Sigma$. Indeed, from what we have seen above, any minimizing sequence is bounded in $L^{2}\left(\mathbf{R}^{d}\right)$, and therefore in $\Sigma$ since $\delta<0$. The Lagrange multiplier $\mu$ associated to this problem is such that

$$
H \psi-\nu \psi=\mu|\psi|^{4 / d} \psi
$$

The scalar product with $\psi$ yields $\mu<0$, since $\delta<0$ :

$$
\nu \psi=H \psi+|\mu||\psi|^{4 / d} \psi .
$$

The function $|\mu|^{d / 4} \psi(\neq 0)$ solves (3.1).

Second case: $\nu<d / 2$.

We show that $0<\delta<\infty$. The finiteness of $\delta$ is obvious, and we recall that the uncertainty principle yields

$$
I(\psi) \geqslant \frac{1}{2}\left(\frac{d}{2}-\nu\right)\|\psi\|_{L^{2}}^{2}>0 .
$$

Assume that $\delta=0$ : we can find a minimizing sequence $\psi_{n} \in M$ such that $\psi_{n} \rightarrow 0$ in $L^{2}$. Therefore,

$$
0 \leftarrow I\left(\psi_{n}\right)=\frac{1}{2}\left\langle H \psi_{n}, \psi_{n}\right\rangle+o(1),
$$


and $\psi_{n} \rightarrow 0$ in $\Sigma$. This implies $\psi_{n} \rightarrow 0$ in $L^{2+4 / d}$ : this contradicts $\psi_{n} \in M$, and so, $\delta>0$. We see from (3.3) that any minimizing sequence is bounded in $L^{2}$, and thereby in $\Sigma$. Up to a subsequence, such a sequence converges weakly in $\Sigma$, and strongly in $L^{2} \cap L^{2+4 / d}$, to a function $\psi \in M$ which verifies

$$
(H-\nu) \psi=\mu|\psi|^{4 / d} \psi \text {. }
$$

Since $\delta>0$, taking the scalar product with $\psi$ in the above equation shows that the Lagrange multiplier $\mu$ is positive. The function $\mu^{d / 4} \psi$ then solves (3.2), hence the proposition.

\section{End of the proof of Theorem 1.8}

Recall that for the existence part of Theorem 1.8, we apply the second point of Proposition 3.1 with the family (for $\theta \in[0,2 \pi[$ )

$$
\left(\nu_{j}\right)_{j \geqslant 1}=\left\{\frac{d}{2}-2 j-\frac{\theta}{\pi}, j \in \mathbf{N} \backslash\{0\}\right\} .
$$

Denote by $\left(\phi_{j}\right)_{j \geqslant 1}$ a corresponding family of even, nontrivial solutions to (3.2). The fact that this family is unbounded in $H^{1}\left(\mathbf{R}^{d}\right)$ follows by taking the scalar product of (3.2) with $\phi_{j}$, and invoking the Gagliardo-Nirenberg inequality (1.11):

$$
\left\langle\left(H-\nu_{j}\right) \phi_{j}, \phi_{j}\right\rangle=\int_{\mathbf{R}^{d}}\left|\phi_{j}(x)\right|^{2+4 / d} d x \leqslant \frac{d+2}{2 d\|Q\|_{L^{2}}^{4 / d}}\left\|\phi_{j}\right\|_{L^{2}}^{4 / d}\left\|\nabla \phi_{j}\right\|_{L^{2}}^{2} .
$$

This reads:

$$
\frac{d+2}{2 d\|Q\|_{L^{2}}^{4 / d}}\left\|\phi_{j}\right\|_{L^{2}}^{4 / d}\left\|\nabla \phi_{j}\right\|_{L^{2}}^{2} \geqslant \frac{1}{2}\left\|\nabla \phi_{j}\right\|_{L^{2}}^{2}+\frac{1}{2}\left\|x \phi_{j}\right\|_{L^{2}}^{2}+\left(2 j-\frac{d}{2}+\frac{\theta}{\pi}\right)\left\|\phi_{j}\right\|_{L^{2}}^{2} .
$$

We have directly, for $2 j>d / 2-\theta / \pi$,

$$
\left\|\phi_{j}\right\|_{L^{2}}^{4 / d}>\frac{d}{d+2}\|Q\|_{L^{2}}^{4 / d}
$$

Therefore,

$$
\left\|\phi_{j}\right\|_{L^{2}}^{4 / d}\left\|\nabla \phi_{j}\right\|_{L^{2}}^{2} \geqslant \frac{2 d}{d+2}\|Q\|_{L^{2}}^{4 / d}\left(2 j-\frac{d}{2}+\frac{\theta}{\pi}\right)\left\|\phi_{j}\right\|_{L^{2}}^{2} \geqslant C\left(2 j-\frac{d}{2}+\frac{\theta}{\pi}\right) .
$$

The last inequality shows that $\left(\phi_{j}\right)_{j \geqslant 1}$ is unbounded in $H^{1}\left(\mathbf{R}^{d}\right)$. The first inequality shows that if $d \geqslant 2$, then

$$
\left\|\nabla \phi_{j}\right\|_{L^{2}}^{2} \gtrsim j
$$

In view of Lemma 2.9, the following result completes the proof of Theorem 1.8.

Proposition 4.1. Let $\nu<d / 2$, and $v(t, x)=e^{-i \nu t} \psi(x)$, where $\psi$ solves (3.2). There exists $\varepsilon>0$ such that if $\phi \in \Sigma$ satisfies $\|v(-\pi / 2, \cdot)-\phi\|_{L^{2}}<\varepsilon$, then the solution $\widetilde{v}$ to the initial value problem

$$
i \partial_{t} \widetilde{v}+\frac{1}{2} \Delta \widetilde{v}=\frac{|x|^{2}}{2} \widetilde{v}-|\widetilde{v}|^{4 / d} \widetilde{v} \quad ; \quad \widetilde{v}_{\mid t=-\pi / 2}=\phi
$$

is such that $\widetilde{v} \in C([-\pi / 2, \pi / 2] ; \Sigma)$. 
Proof. For $\phi \in \Sigma$, the local existence of a solution $\widetilde{v}$ in $\Sigma$ is standard; see e.g. $[5,10]$. We prove that if $\varepsilon$ is sufficiently small, then this solution cannot blow-up on the time interval $[-\pi / 2, \pi / 2]$. The analysis in [5] yields, since $v \in$ $C([-\pi / 2, \pi / 2] ; \Sigma)$ :

$$
v, x v, \nabla v \in L^{p}\left([-\pi / 2, \pi / 2] ; L^{q}\left(\mathbf{R}^{d}\right)\right), \quad \forall(p, q) \text { admissible. }
$$

Consider the function $w=v-\widetilde{v}$. It solves

$$
i \partial_{t} w=H w+g(v+w)-g(v) \quad ; \quad w_{\mid t=-\pi / 2}=v-\widetilde{v}_{\mid t=-\pi / 2},
$$

where we have denoted $g(z)=|z|^{4 / d} z$. To prove the proposition, it suffices to show that $w \in C([-\pi / 2, \pi / 2] ; \Sigma)$. Let $t \in[-\pi / 2, \pi / 2]$, and denote $D_{t}=[-\pi / 2, t] \times \mathbf{R}^{d}$. Strichartz estimates with the admissible pair $(2+4 / d, 2+4 / d)$ yield, along with Hölder inequality:

$$
\begin{aligned}
\|w\|_{L^{2+4 / d}\left(D_{t}\right)} \leqslant C\left\|v-\widetilde{v}_{\mid t=-\pi / 2}\right\|_{L^{2}} & \\
& +C\left(\|w\|_{L^{2+4 / d}\left(D_{t}\right)}^{4 / d}+\|v\|_{L^{2+4 / d}\left(D_{t}\right)}^{4 / d}\right)\|w\|_{L^{2+4 / d}\left(D_{t}\right)} .
\end{aligned}
$$

Note that the constant $C$ can be chosen independent of $t \in[-\pi / 2, \pi / 2]$, by considering $I=[-\pi / 2, \pi / 2]$ in Lemma 2.2. By splitting $I$ into a finite number of intervals $I_{j}$ such that

$$
C\|v\|_{L^{2+4 / d}\left(I_{j} \times \mathbf{R}^{d}\right)}^{4 / d} \leqslant \frac{1}{2},
$$

and repeating the same arguments finitely many times, we see that there exists $C_{0}$ such that

$$
\|w\|_{L^{2+4 / d}\left(D_{t}\right)} \leqslant C_{0}\left\|v-\widetilde{v}_{\mid t=-\pi / 2}\right\|_{L^{2}}+C_{0}\|w\|_{L^{2+4 / d}\left(D_{t}\right)}^{4 / d+1}
$$

for all $t \in I$ (this is essentially Gronwall lemma on a finite time interval). Therefore, choosing $\left\|v-\widetilde{v}_{\mid t=-\pi / 2}\right\|_{L^{2}}$ sufficiently small, a bootstrap argument shows that $w \in$ $L^{2+4 / d}\left(I \times \mathbf{R}^{d}\right)$.

Since the operators $x$ and $\nabla$ do not commute with $U_{H}$, we may introduce the operators

$$
J(t)=x \sin t-i \cos t \nabla \quad ; \quad K(t)=x \cos t+i \sin t \nabla .
$$

These operators commute with $U_{H}$, act on gauge invariant nonlinearities like derivatives, and satisfy the pointwise property

$$
|J(t) f|^{2}+|K(t) f|^{2}=|x f|^{2}+|\nabla f|^{2} .
$$

We refer to [5] for more details. Applying the operators $J$ and $K$ to (4.2), Strichartz and Hölder inequalities yield

$$
\begin{gathered}
\|J w\|_{L^{2+4 / d}\left(D_{t}\right)}+\|K w\|_{L^{2+4 / d}\left(D_{t}\right)} \leqslant C\left\|v-\widetilde{v}_{\mid t=-\pi / 2}\right\|_{\Sigma} \\
+C\left(\|w\|_{L^{2+4 / d}\left(D_{t}\right)}^{4 / d}+\|v\|_{L^{2+4 / d}\left(D_{t}\right)}^{4 / d}\right)\left(\|J v\|_{L^{2+4 / d}\left(D_{t}\right)}+\|K v\|_{L^{2+4 / d}\left(D_{t}\right)}\right) \\
+C\left(\|r\|_{L^{2+4 / d}\left(D_{t}\right)}^{4 / d}+\|v\|_{L^{2+4 / d}\left(D_{t}\right)}^{4 / d}\right)\left(\|J w\|_{L^{2+4 / d}\left(D_{t}\right)}+\|K w\|_{L^{2+4 / d}\left(D_{t}\right)}\right) .
\end{gathered}
$$

Splitting $I$ into intervals where

$$
C\left(\|w\|_{L^{2+4 / d}\left(I_{j} \times \mathbf{R}^{d}\right)}^{4 / d}+\|v\|_{L^{2+4 / d}\left(I_{j} \times \mathbf{R}^{d}\right)}^{4 / d}\right) \leqslant \frac{1}{2},
$$

we infer that $J w, K w \in L^{2+4 / d}\left(I \times \mathbf{R}^{d}\right)$. Applying Strichartz inequality with now $\left(p_{1}, q_{1}\right)=(\infty, 2)$ and $\left(p_{2}, q_{2}\right)=(2+4 / d, 2+4 / d)$, we see that $w, J w, K w \in$ 
$L^{\infty}\left(I ; L^{2}\left(\mathbf{R}^{d}\right)\right)$, hence $w, x w, \nabla w \in L^{\infty}\left(I ; L^{2}\left(\mathbf{R}^{d}\right)\right)$ from (4.3). The results in [5] imply that $w \in C([-\pi / 2, \pi / 2] ; \Sigma)$.

\section{Appendix A. Profile decomposition and nonlinear superposition}

Consider first the energy-critical nonlinear Schrödinger equation in space dimension $d=3$ :

$$
i \partial_{t} u+\frac{1}{2} \Delta u=|u|^{4} u \quad ; \quad x \in \mathbf{R}^{3} .
$$

Before stating the results we want to recall from [21], introduce a definition:

Definition A.1. If $\left(h_{j}^{\varepsilon}, t_{j}^{\varepsilon}, x_{j}^{\varepsilon}\right)_{j \in \mathbf{N}}$ is a family of sequences in $\mathbf{R}_{+} \backslash\{0\} \times \mathbf{R} \times \mathbf{R}^{3}$, then we say that $\left(h_{j}^{\varepsilon}, t_{j}^{\varepsilon}, x_{j}^{\varepsilon}\right)_{j \in \mathbf{N}}$ is an orthogonal family if

$$
\limsup _{\varepsilon \rightarrow 0}\left(\frac{h_{j}^{\varepsilon}}{h_{k}^{\varepsilon}}+\frac{h_{k}^{\varepsilon}}{h_{j}^{\varepsilon}}+\frac{\left|t_{j}^{\varepsilon}-t_{k}^{\varepsilon}\right|}{\left(h_{j}^{\varepsilon}\right)^{2}}+\left|\frac{x_{j}^{\varepsilon}-x_{k}^{\varepsilon}}{h_{j}^{\varepsilon}}\right|\right)=\infty, \quad \forall j \neq k .
$$

The main two results in [21], which we recall below, are the Schrödinger analogues to the results in [1] for the wave equation (1.8).

Theorem A.2 (Theorem 1.6 in [21]). Let $\left(\phi^{\varepsilon}\right)_{0<\varepsilon \leqslant 1}$ be a bounded family in $\dot{H}^{1}\left(\mathbf{R}^{3}\right)$. Let $u_{\text {lin }}^{\varepsilon}=e^{i \frac{t}{2} \Delta} \phi^{\varepsilon}$. Then, up to a subsequence (still denoted by $u_{\text {lin }}^{\varepsilon}$ ), there exist a family $\left(h_{j}^{\varepsilon}\right)_{j \geqslant 1}$ of positive numbers, a family $\left(t_{j}^{\varepsilon}, x_{j}^{\varepsilon}\right)_{j \geqslant 1}$ of vectors in $\mathbf{R} \times \mathbf{R}^{3}$, and a family $\left(V_{j}\right)_{j} \geqslant 1$ of solutions to

$$
i \partial_{t} V+\frac{1}{2} \Delta V=0
$$

such that:

- $\left(h_{j}^{\varepsilon}, t_{j}^{\varepsilon}, x_{j}^{\varepsilon}\right)_{j \in \mathbf{N}}$ is an orthogonal family.

- For every $\ell \geqslant 1$,

$$
u_{\operatorname{lin}}^{\varepsilon}(t, x)=\sum_{j=1}^{\ell} \frac{1}{\sqrt{h_{j}^{\varepsilon}}} V_{j}\left(\frac{t-t_{j}^{\varepsilon}}{\left(h_{j}^{\varepsilon}\right)^{2}}, \frac{x-x_{j}^{\varepsilon}}{h_{j}^{\varepsilon}}\right)+w_{\ell}^{\varepsilon}(t, x),
$$

with

$$
\limsup _{\varepsilon \rightarrow 0}\left\|w_{\ell}^{\varepsilon}\right\|_{L^{q}\left(\mathbf{R} ; L^{r}\left(\mathbf{R}^{3}\right)\right)} \underset{\ell \rightarrow \infty}{\longrightarrow} 0,
$$

for every pair $(q, r)$ with $6 \leqslant r<\infty$ and $2 / q+3 / r=1 / 2$.

In [21], we find, since every $\dot{H}^{1}$ solution to (A.1) is global in time [14]:

Theorem A.3 (From [21] and [14]). Under the same assumptions as in Theorem A.2, consider the solutions to

$$
i \partial_{t} u^{\varepsilon}+\frac{1}{2} \Delta u^{\varepsilon}=\left|u^{\varepsilon}\right|^{4} u^{\varepsilon} ; \quad u^{\varepsilon}(0, x)=\phi^{\varepsilon}(x),
$$

associated with the subsequence of Theorem A.2. Then

$$
u^{\varepsilon}(t, x)=\sum_{j=1}^{\ell} \frac{1}{\sqrt{h_{j}^{\varepsilon}}} U_{j}\left(\frac{t-t_{j}^{\varepsilon}}{\left(h_{j}^{\varepsilon}\right)^{2}}, \frac{x-x_{j}^{\varepsilon}}{h_{j}^{\varepsilon}}\right)+w_{\ell}^{\varepsilon}(t, x)+r_{\ell}^{\varepsilon}(t, x),
$$

with

$$
\limsup _{\varepsilon \rightarrow 0}\left(\left\|\nabla r_{\ell}^{\varepsilon}\right\|_{L^{\infty}\left(\mathbf{R} ; L^{2}\left(\mathbf{R}^{3}\right)\right)}+\left\|r_{\ell}^{\varepsilon}\right\|_{L^{10}\left(\mathbf{R}^{4}\right)}+\left\|\nabla r_{\ell}^{\varepsilon}\right\|_{L^{10 / 3}\left(\mathbf{R}^{4}\right)}\right) \underset{\ell \rightarrow \infty}{\longrightarrow} 0
$$


where $h_{j}^{\varepsilon}, t_{j}^{\varepsilon}, x_{j}^{\varepsilon}, w_{\ell}^{\varepsilon}$ are as in Theorem A.2, and the nonlinear profiles $U_{j}$ are given by:

$$
i \partial_{t} U_{j}+\frac{1}{2} \Delta U_{j}=\left|U_{j}\right|^{4} U_{j} \quad ; \quad\left\|\nabla\left(U_{j}-V_{j}\right)\left(-\frac{t_{j}^{\varepsilon}}{\left(h_{j}^{\varepsilon}\right)^{2}}\right)\right\|_{L^{2}\left(\mathbf{R}^{3}\right)} \underset{\varepsilon \rightarrow 0}{\longrightarrow} 0 .
$$

Note that according to the limit of $t_{j}^{\varepsilon} /\left(h_{j}^{\varepsilon}\right)^{2}$ in $[-\infty,+\infty]$, the profile $U_{j}$ is defined either by a Cauchy data, or by an asymptotic state. Roughly speaking, the contribution of $w_{\ell}^{\varepsilon}$ is linear, since this function is the same as in the linear profile decomposition of Theorem A.2, while $r_{\ell}^{\varepsilon}$ is asymptotically small (thus linear) as $\ell \rightarrow \infty$. All in all, (leading order) nonlinear effects are measured through the nonlinear profiles $U_{j}$. The orthogonality property shows that the interactions of the scaled profiles are negligible in the limit $\varepsilon \rightarrow 0$. The large time behavior of $u^{\varepsilon}$ is given, asymptotically as $\varepsilon \rightarrow 0$, by the superposition of the large time behavior of the scaled nonlinear profiles. Since every profile $U_{j}$ possesses asymptotic states, we see that $S$ acts on each profile separately (as $\varepsilon \rightarrow 0$ ).

In the $L^{2}$-critical case (1.1), the profile decomposition at the $L^{2}$ level is not merely a recasting of its $\dot{H}^{1}$ counterpart, because Galilean invariance must be taken into account. A profile decomposition was introduced in [28] in the case $d=2$, then generalized to the case $d \leqslant 2$ in [8], in such a way that the improved Strichartz estimates in [2] yield a profile decomposition in $L^{2}\left(\mathbf{R}^{d}\right)$ associated to solutions of (1.1) for all $d \geqslant 1$. Due the existence of an extra invariance, we modify the notion of orthogonal scales and cores:

Definition A.4. If $\left(h_{j}^{\varepsilon}, t_{j}^{\varepsilon}, x_{j}^{\varepsilon}, \xi_{j}^{\varepsilon}\right)_{j \in \mathbf{N}}$ is a family of sequences in $\mathbf{R}_{+} \backslash\{0\} \times$ $\mathbf{R} \times \mathbf{R}^{d} \times \mathbf{R}^{d}$, then we say that $\left(h_{j}^{\varepsilon}, t_{j}^{\varepsilon}, x_{j}^{\varepsilon}, \xi_{j}^{\varepsilon}\right)_{j \in \mathbf{N}}$ is an orthogonal family if

$$
\limsup _{\varepsilon \rightarrow 0}\left(\frac{h_{j}^{\varepsilon}}{h_{k}^{\varepsilon}}+\frac{h_{k}^{\varepsilon}}{h_{j}^{\varepsilon}}+\frac{\left|t_{j}^{\varepsilon}-t_{k}^{\varepsilon}\right|}{\left(h_{j}^{\varepsilon}\right)^{2}}+\left|\frac{x_{j}^{\varepsilon}-x_{k}^{\varepsilon}}{h_{j}^{\varepsilon}}+\frac{t_{j}^{\varepsilon} \xi_{j}^{\varepsilon}-t_{k}^{\varepsilon} \xi_{k}^{\varepsilon}}{h_{j}^{\varepsilon}}\right|\right)=\infty, \quad \forall j \neq k .
$$

Theorem A.5 (From $[28,8,2])$. Let $d \geqslant 1$ and $\left(\phi^{\varepsilon}\right)_{0<\varepsilon \leqslant 1}$ be a bounded family in $L^{2}\left(\mathbf{R}^{d}\right)$. Up to extracting a subsequence, we have:

i) There exist an orthogonal family $\left(h_{j}^{\varepsilon}, t_{j}^{\varepsilon}, x_{j}^{\varepsilon}, \xi_{j}^{\varepsilon}\right)_{j \in \mathbf{N}}$ in $\mathbf{R}_{+} \backslash\{0\} \times \mathbf{R} \times \mathbf{R}^{d} \times \mathbf{R}^{d}$, and a family $\left(\phi_{j}\right)_{j \in \mathbf{N}}$ bounded in $L^{2}\left(\mathbf{R}^{d}\right)$, such that for every $\ell \geqslant 1$,

$$
e^{i \frac{t}{2} \Delta} \phi^{\varepsilon}=\sum_{j=1}^{\ell} P_{j}^{\varepsilon}\left(\phi_{j}\right)(t, x)+r_{\ell}^{\varepsilon}(t, x)
$$

$$
\begin{gathered}
\text { where } \quad P_{j}^{\varepsilon}\left(\phi_{j}\right)(t, x)=e^{i x \cdot \xi_{j}^{\varepsilon}-i \frac{t}{2}\left|\xi_{j}^{\varepsilon}\right|^{2}} \frac{1}{\left(h_{j}^{\varepsilon}\right)^{d / 2}} V_{j}\left(\frac{t-t_{j}^{\varepsilon}}{\left(h_{j}^{\varepsilon}\right)^{2}} \frac{x-x_{j}^{\varepsilon}-t \xi_{j}^{\varepsilon}}{h_{j}^{\varepsilon}}\right), \\
\text { with } \quad V_{j}(t)=e^{i \frac{t}{2} \Delta} \phi_{j}, \quad \text { and } \quad \limsup _{\varepsilon \rightarrow 0}\left\|r_{\ell}^{\varepsilon}\right\|_{L^{2+4 / d}\left(\mathbf{R} \times \mathbf{R}^{d}\right)} \underset{\ell \rightarrow+\infty}{\longrightarrow} 0 .
\end{gathered}
$$

Furthermore, for every $\ell \geqslant 1$, we have

$$
\left\|\phi^{\varepsilon}\right\|_{L^{2}\left(\mathbf{R}^{d}\right)}^{2}=\sum_{j=1}^{\ell}\left\|\phi_{j}\right\|_{L^{2}\left(\mathbf{R}^{d}\right)}^{2}+\left\|r_{\ell}^{\varepsilon}\right\|_{L^{2}\left(\mathbf{R}^{d}\right)}^{2}+o(1) \quad \text { as } \varepsilon \rightarrow 0 .
$$

ii) If in addition the family $\left(\phi^{\varepsilon}\right)_{0<\varepsilon \leqslant 1}$ is bounded in $H^{1}\left(\mathbf{R}^{d}\right)$, or more generally if

$$
\limsup _{\varepsilon \rightarrow 0} \int_{|\xi|>R}\left|\widehat{\phi}^{\varepsilon}(\xi)\right|^{2} d \xi \rightarrow 0 \quad \text { as } R \rightarrow+\infty
$$


then for every $j \geqslant 1, h_{j}^{\varepsilon} \geqslant 1$, and $\left(\xi_{j}^{\varepsilon}\right)_{\varepsilon}$ is bounded, $\left|\xi_{j}^{\varepsilon}\right| \leqslant C_{j}$.

Contrary to the case of (A.1), the global existence of solutions to (1.1) in the critical space $\left(L^{2}\right)$ is not known so far, hence a slightly intricate statement (as in [21] for the $\dot{H}^{1}$ case, written at a time where the global existence for (A.1) was not known):

Theorem A.6 (From $[28,8,2])$. Under the same assumptions as in Theorem A.5, consider the solutions to

$$
i \partial_{t} u^{\varepsilon}+\frac{1}{2} \Delta u^{\varepsilon}=\left|u^{\varepsilon}\right|^{4 / d} u^{\varepsilon} \quad ; \quad u^{\varepsilon}(0, x)=\phi^{\varepsilon}(x),
$$

associated with the subsequence of Theorem A.5. Consider the solution $U_{j}$ to (1.1) such that

$$
\left\|\left(U_{j}-V_{j}\right)\left(\frac{-t^{\varepsilon}}{\left(h^{\varepsilon}\right)^{2}}\right)\right\|_{L^{2}\left(\mathbf{R}^{n}\right)} \underset{\varepsilon \rightarrow 0}{\longrightarrow} 0 .
$$

Let $I^{\varepsilon} \subset \mathbf{R}$ be a family of open intervals containing the origin. The following statements are equivalent:

(i) For every $j \geqslant 1$, we have

$$
\limsup _{\varepsilon \rightarrow 0}\left\|U_{j}\right\|_{L^{2+4 / d}\left(I_{j}^{\varepsilon} \times \mathbf{R}^{d}\right)}<+\infty, \quad \text { where } I_{j}^{\varepsilon}:=\left(h_{j}^{\varepsilon}\right)^{-2}\left(I^{\varepsilon}-t_{j}^{\varepsilon}\right) \text {. }
$$

(ii) $\limsup _{\varepsilon \rightarrow 0}\left\|u^{\varepsilon}\right\|_{L^{2+4 / d}\left(I^{\varepsilon} \times \mathbf{R}^{d}\right)}<+\infty$.

Moreover, if (i) or (ii) holds, then $u^{\varepsilon}=\sum_{j=1}^{\ell} N_{j}^{\varepsilon}\left(\phi_{j}\right)+r_{\ell}^{\varepsilon}+\rho_{\ell}^{\varepsilon}$, where $r_{\ell}^{\varepsilon}$ is given by Theorem A.5, and:

$$
\begin{array}{r}
\limsup _{\varepsilon \rightarrow 0}\left(\left\|\rho_{\ell}^{\varepsilon}\right\|_{L^{2+4 / d}\left(I^{\varepsilon} \times \mathbf{R}^{d}\right)}+\left\|\rho_{\ell}^{\varepsilon}\right\|_{L^{\infty}\left(I^{\varepsilon} ; L^{2}\left(\mathbf{R}^{d}\right)\right)}\right) \underset{\ell \rightarrow+\infty}{\longrightarrow} 0, \\
N_{j}^{\varepsilon}\left(\phi_{j}\right)(t, x)=e^{i x \cdot \xi_{j}^{\varepsilon}-i \frac{t}{2}\left|\xi_{j}^{\varepsilon}\right|^{2}} \frac{1}{\left(h_{j}^{\varepsilon}\right)^{d / 2}} U_{j}\left(\frac{t-t_{j}^{\varepsilon}}{\left(h_{j}^{\varepsilon}\right)^{2}} \frac{x-x_{j}^{\varepsilon}-t \xi_{j}^{\varepsilon}}{h_{j}^{\varepsilon}}\right) .
\end{array}
$$

If, as expected, one has $I_{j}^{\varepsilon}=I^{\varepsilon}=\mathbf{R}$ for all $j$, then this result is the exact analogue of Theorem A.3.

\section{References}

[1] H. Bahouri and P. Gérard, High frequency approximation of solutions to critical nonlinear wave equations, Amer. J. Math. 121 (1999), no. 1, 131-175.

[2] P. Bégout and A. Vargas, Mass concentration phenomena for the $L^{2}$-critical nonlinear Schrödinger equation, Trans. Amer. Math. Soc. 359 (2007), no. 11, 5257-5282.

[3] H. Berestycki and P.-L. Lions, Nonlinear scalar field equations. I. Existence of a ground state, Arch. Rational Mech. Anal. 82 (1983), no. 4, 313-345.

[4] R. Carles, Critical nonlinear Schrödinger equations with and without harmonic potential, Math. Models Methods Appl. Sci. 12 (2002), no. 10, 1513-1523.

[5] R. Carles, Remarks on nonlinear Schrödinger equations with harmonic potential, Ann. Henri Poincaré 3 (2002), no. 4, 757-772.

[6] R. Carles, Semi-classical analysis for nonlinear Schrödinger equations, World Scientific Publishing Co. Pte. Ltd., Hackensack, NJ, 2008.

[7] R. Carles and I. Gallagher, Analyticity of the scattering operator for semilinear dispersive equations, Comm. Math. Phys. 286 (2009), no. 3, 1181-1209.

[8] R. Carles and S. Keraani, On the role of quadratic oscillations in nonlinear Schrödinger equations II. The $L^{2}$-critical case, Trans. Amer. Math. Soc. 359 (2007), no. 1, 33-62. 
[9] R. Carles and T. Ozawa, On the wave operators for the critical nonlinear Schrödinger equation critical nonlinear Schrödinger equation, Math. Res. Lett. 15 (2008), no. 1, 185-195.

[10] T. Cazenave, Semilinear Schrödinger equations, Courant Lecture Notes in Mathematics, vol. 10, New York University Courant Institute of Mathematical Sciences, New York, 2003.

[11] T. Cazenave and F. Weissler, Some remarks on the nonlinear Schrödinger equation in the critical case, Lect. Notes in Math., vol. 1394, Springer-Verlag, Berlin, 1989, pp. 18-29.

[12] T. Cazenave and F. Weissler, Rapidly decaying solutions of the nonlinear Schrödinger equation, Comm. Math. Phys. 147 (1992), 75-100.

[13] S. Coleman, V. Glaser, and A. Martin, Action minima among solutions to a class of Euclidean scalar field equations, Comm. Math. Phys. 58 (1978), no. 2, 211-221.

[14] J. Colliander, M. Keel, G. Staffilani, H. Takaoka, and T. Tao, Global well-posedness and scattering for the energy-critical nonlinear Schrödinger equation in $\mathbb{R}^{3}$, Ann. of Math. (2) 167 (2008), no. 3, 767-865.

[15] P. Gérard, Oscillations and concentration effects in semilinear dispersive wave equations, J. Funct. Anal. 141 (1996), no. 1, 60-98.

[16] P. Gérard, Description du défaut de compacité de l'injection de Sobolev, ESAIM Control Optim. Calc. Var. 3 (1998), 213-233.

[17] J. Ginibre and G. Velo, On a class of nonlinear Schrödinger equations. II Scattering theory, general case, J. Funct. Anal. 32 (1979), 33-71.

[18] N. Hayashi and Y. Tsutsumi, Remarks on the scattering problem for nonlinear Schrödinger equations, Differential equations and mathematical physics (Birmingham, Ala., 1986), Lectures Notes in Math., vol. 1285, Springer, Berlin, 1987, pp. 162-168.

[19] T. Hmidi and S. Keraani, Remarks on the blowup for the $L^{2}$-critical nonlinear Schrödinger equations, SIAM J. Math. Anal. 38 (2006), no. 4, 1035-1047.

[20] O. Kavian and F. Weissler, Self-similar solutions of the pseudo-conformally invariant nonlinear Schrödinger equation, Michigan Math. J. 41 (1994), no. 1, 151-173.

[21] S. Keraani, On the defect of compactness for the Strichartz estimates of the Schrödinger equations, J. Diff. Eq. 175 (2001), no. 2, 353-392.

[22] S. Keraani, On the blow up phenomenon of the critical nonlinear Schrödinger equation, J. Funct. Anal. 235 (2006), no. 1, 171-192.

[23] G. A. Kerimov, Non-spherically symmetric transparent potentials for the three-dimensional Schrödinger equation, J. Phys. A: Math. Theor. 40 (2007), 11607-11615.

[24] R. Killip, T. Tao, and M. Visan, The cubic nonlinear Schrödinger equation in two dimensions with radial data, J. Eur. Math. Soc. (JEMS), to appear.

[25] R. Killip, M. Visan, and X. Zhang, The mass-critical nonlinear Schrödinger equation with radial data in dimensions three and higher, archived as arXiv:0708.0849.

[26] L. D. Landau and E. M. Lifshitz, Quantum mechanics: non-relativistic theory. Course of Theoretical Physics, Vol. 3, Addison-Wesley Series in Advanced Physics, Pergamon Press Ltd., London-Paris, 1958, Translated from the Russian by J. B. Sykes and J. S. Bell.

[27] F. Merle and Y. Tsustumi, $L^{2}$-concentration of blow-up solutions for the nonlinear Schrödinger equation with the critical power nonlinearity, J. Diff. Eq. 84 (1990), 205-214.

[28] F. Merle and L. Vega, Compactness at blow-up time for $L^{2}$ solutions of the critical nonlinear Schrödinger equation in 2D, Internat. Math. Res. Notices (1998), no. 8, 399-425.

[29] G. Métivier and S. Schochet, Trilinear resonant interactions of semilinear hyperbolic waves, Duke Math. J. 95 (1998), no. 2, 241-304.

[30] K. Nakanishi and T. Ozawa, Remarks on scattering for nonlinear Schrödinger equations, NoDEA Nonlinear Differential Equations Appl. 9 (2002), no. 1, 45-68.

[31] L. Nirenberg, Topics in nonlinear functional analysis, Courant Lecture Notes in Mathematics, vol. 6, New York University Courant Institute of Mathematical Sciences, New York, 2001, Chapter 6 by E. Zehnder, Notes by R. A. Artino, Revised reprint of the 1974 original.

[32] J. Rauch, Partial differential equations, Graduate Texts in Math., vol. 128, Springer-Verlag, New York, 1991.

[33] H. A. Rose and M. I. Weinstein, On the bound states of the nonlinear Schrödinger equation with a linear potential, Phys. D 30 (1988), no. 1-2, 207-218.

[34] A. V. Rybin, G. G. Varzugin, M. Lindberg, J. Timonen, and R. K. Bullough, Similarity solutions and collapse in the attractive Gross-Pitaevskii equation, Phys. Rev. E (3) 62 (2000), no. 5, part A, 6224-6228. 
[35] T. Tao, A pseudoconformal compactification of the nonlinear Schrödinger equation and applications, archived as arXiv:math/0606254.

[36] T. Tao, Nonlinear dispersive equations, CBMS Regional Conference Series in Mathematics, vol. 106, Published for the Conference Board of the Mathematical Sciences, Washington, DC, 2006, Local and global analysis.

[37] T. Tao, M. Visan, and X. Zhang, Global well-posedness and scattering for the mass-critical nonlinear Schrödinger equation for radial data in high dimensions, Duke Math. J. 140 (2007), no. 1, 165-202.

[38] Y. Tsutsumi, Scattering problem for nonlinear Schrödinger equations, Ann. Inst. H. Poincaré Phys. Théor. 43 (1985), no. 3, 321-347.

[39] N. Tzirakis, Mass concentration phenomenon for the quintic nonlinear Schrödinger equation in one dimension, SIAM J. Math. Anal. 37 (2006), no. 6, 1923-1946.

[40] M. Visan and X. Zhang, On the blowup for the $L^{2}$-critical focusing nonlinear Schrödinger equation in higher dimensions below the energy class, SIAM J. Math. Anal. 39 (2007), no. 1, $34-56$.

[41] M. I. Weinstein, Nonlinear Schrödinger equations and sharp interpolation estimates, Comm. Math. Phys. 87 (1982/83), no. 4, 567-576.

Université Montpellier 2 Mathématiques, CC051 34095 Montpellier, France

CNRS, UMR 514934095 Montpellier, France

E-mail address: Remi.Carles@math.cnrs.fr 